\title{
Wind from the black-hole accretion disk driving a molecular outflow in an active galaxy
}

\author{
F. Tombesi ${ }^{1,2}$, M. Meléndez ${ }^{2}$, S. Veilleux ${ }^{2,3}$, J. N. Reeves ${ }^{4,5}$, E. González-Alfonso ${ }^{6}$ \& C. S. Reynolds ${ }^{2,3}$
}

Powerful winds driven by active galactic nuclei are often thought to affect the evolution of both supermassive black holes and their host galaxies, quenching star formation and explaining the close relationship between black holes and galaxies ${ }^{1,2}$. Recent observations of large-scale molecular outflows ${ }^{3-8}$ in ultraluminous infrared galaxies support this quasar-feedback idea, because they directly trace the gas from which stars form. Theoretical models ${ }^{9-12}$ suggest that these outflows originate as energy-conserving flows driven by fast accretiondisk winds. Proposed connections between large-scale molecular outflows and accretion-disk activity in ultraluminous galaxies were incomplete ${ }^{3-8}$ because no accretion-disk wind had been detected. Conversely, studies of powerful accretion-disk winds have until now focused only on X-ray observations of local Seyfert galaxies ${ }^{13,14}$ and a few higher-redshift quasars ${ }^{15-19}$. Here we report observations of a powerful accretion-disk wind with a mildly relativistic velocity (a quarter that of light) in the X-ray spectrum of IRAS F11119+3257, a nearby (redshift 0.189) optically classified type 1 ultraluminous infrared galaxy hosting a powerful molecular outflow ${ }^{6}$. The active galactic nucleus is responsible for about 80 per cent of the emission, with a quasar-like luminosity ${ }^{6}$ of $1.5 \times 10^{46}$ ergs per second. The energetics of these two types of wide-angle outflows is consistent with the energyconserving mechanism $^{9-12}$ that is the basis of the quasar feedback ${ }^{1}$ in active galactic nuclei that lack powerful radio jets (such jets are an alternative way to drive molecular outflows).

The mass ${ }^{20}$ of the central supermassive black hole in IRAS F11119+ 3257 is estimated to be $M_{\mathrm{BH}} \approx 1.6 \times 10^{7} M_{\odot}$, where $M_{\odot}$ is the solar mass. The resulting Eddington ratio is $L_{\mathrm{AGN}} / L_{\mathrm{Edd}} \approx 5$, where $L_{\mathrm{AGN}}$ is the luminosity of the active galactic nucleus (AGN), indicating that the source is very likely to be accreting at about its Eddington limit. In the $\mathrm{X}$-ray spectrum it is relatively bright and not strongly affected by neutral absorption ${ }^{21}$. Therefore, it is arguably the best candidate in which to study the highly ionized iron K-band absorbers in this class of object.

IRAS F11119+3257 was the subject of a long (250-ks) observation from the Suzaku X-ray astronomy satellite obtained by our group in May 2013. The spectra of the three separated X-ray imaging spectrometer (XIS) detectors onboard Suzaku independently show a prominent absorption feature at the rest-frame energy of $\sim 9 \mathrm{keV}$ (Fig. 1). The inclusion of a broad Gaussian absorption line in the combined broadband Suzaku XIS and the hard X-ray detector PIN spectrum in the 0.5$25 \mathrm{keV}$ energy band provides a very significant improvement of the fit, corresponding to a statistical confidence level of $6.5 \sigma$ (see Supplementary Information). The absorption line has a rest-frame energy of $E=$ $9.82_{-0.34}^{+0.64} \mathrm{keV}$, a width of $\sigma_{E}=1.67_{-0.44}^{+1.00} \mathrm{keV}$ and an equivalent width of $-1.31_{-0.31}^{+0.40} \mathrm{keV}$. If interpreted as blue-shifted Fe Xxv He $\alpha$ and/or Fe XXVI Lyman $\alpha$ resonant absorption lines, this feature indicates an outflow velocity of about $0.3 c$ (where $c$ is the velocity of light in a vacuum) with respect to the systemic velocity.

We derive a physical characterization of this outflow using a dedicated photoionization absorption model (see Methods). This fast-wind model provides a very good representation of the data, simultaneously taking into account both the broad absorption trough at $E \approx 7-10 \mathrm{keV}$ in the XIS data and the factor of $\sim 2-3$ excess of flux at $E=15-25 \mathrm{keV}$ observed in the PIN data (see Extended Data Fig. 1 and Methods). The column density, ionization and outflow velocity are $N_{\mathrm{H}}=6.4_{-1.3}^{+0.8} \times 10^{24} \mathrm{~cm}^{-2}$, $\log \xi=4.11_{-0.04}^{+0.09} \mathrm{erg} \mathrm{s}^{-1} \mathrm{~cm}$ and $v_{\text {out }, \mathrm{X}}=0.255 \pm 0.011 c$, respectively (see Extended Data Table 1). The wind has a high covering fraction of $C_{\mathrm{F}, \mathrm{X}}>0.85$, a value that is confirmed if we include the possible associated emission in the best-fit model (see Supplementary Information). The wind parameters are consistent overall with the mildly relativistic accretiondisk winds detected in quasars ${ }^{15-19}$ with a comparable luminosity.

Considering the velocity and variability of the wind we estimate a distance from the central supermassive black hole in units of Schwarzschild radii $\left(r_{\mathrm{S}}=2 G M_{\mathrm{BH}} / c^{2}\right)$ (where $G$ is the gravitational constant) of $r \approx$ $(15-900) r_{\mathrm{S}}$, consistent with accretion-disk scales (see Supplementary Information). Conservatively adopting a radius of $15 r_{\mathrm{S}}$, we estimate a mass outflow rate of $\dot{M}_{\text {out }, \mathrm{X}} \approx 1.5 \mathrm{M} \odot \mathrm{yr}^{-1}$. Given the very high luminosity of this active galactic nucleus and the fact that it is accreting at about its Eddington limit, it is very likely that the wind is radiation driven $^{22}$.

The wind's momentum flux $\dot{P}_{\text {out }, \mathrm{X}}=\dot{M}_{\text {out }, \mathrm{X}} v_{\text {out }, \mathrm{X}} \approx 6 \times 10^{35}$ dyne corresponds to $1.3_{-0.9}^{+1.7}$ times that of the AGN radiation $\dot{P}_{\text {rad }}=L_{\mathrm{AGN}} / c$ (see Supplementary Information). This is consistent with multiple

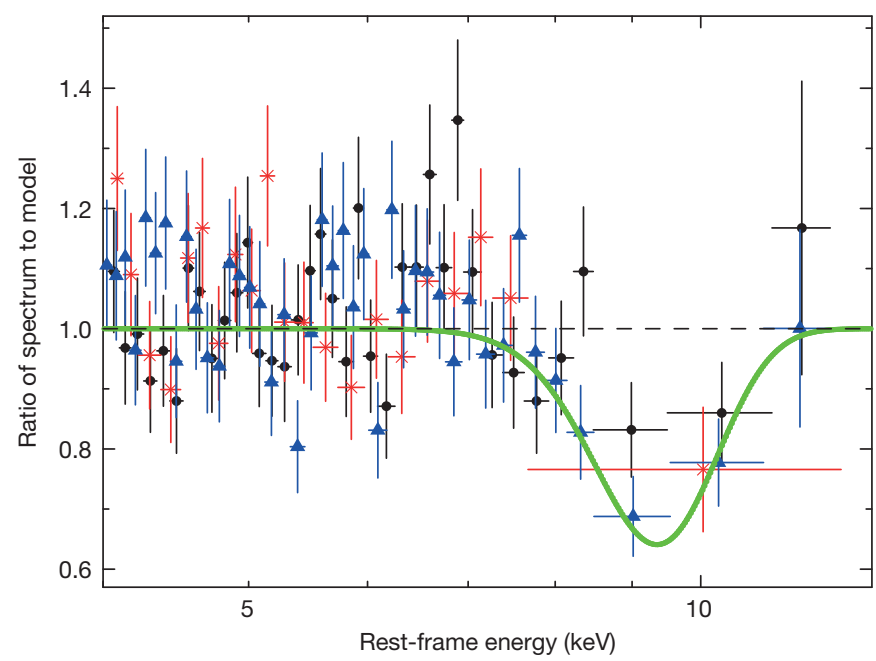

Figure $1 \mid$ Absorption line in the Suzaku spectrum of IRAS F11119+3257. Ratio between the separated spectra of the Suzaku detectors XIS0 (black), XIS1 (red) and XIS3 (blue) and the absorbed power-law continuum model in the $E=4-12 \mathrm{keV}$ energy range. The ratio for the model including the absorption line at the rest-frame energy of $9.82 \mathrm{keV}$ is superimposed in green. The data are binned to a signal-to-noise ratio of $10 \sigma$ for clarity. Errors are at the $1 \sigma$ level.

${ }^{1}$ X-ray Astrophysics Laboratory, NASA/Goddard Space Flight Center, Greenbelt, Maryland 20771, USA. ${ }^{2}$ Department of Astronomy and CRESST, University of Maryland, College Park, Maryland 20742, USA. ${ }^{3}$ Joint Space Science Institute, University of Maryland, College Park, Maryland 20742, USA. ${ }^{4}$ Astrophysics Group, School of Physical and Geographical Sciences, Keele University, Keele, Staffordshire ST5 5BG, UK. ${ }^{5}$ Center for Space Science and Technology, University of Maryland Baltimore County, 1000 Hilltop Circle, Baltimore, Maryland 21250, USA. ${ }^{6}$ Universidad de Alcalá, Departamento de Física y Matemáticas, Campus Universitario, E-28871 Alcalá de Henares, Madrid, Spain. 
scatterings in a high-column-density and wide-angle wind ${ }^{23}$. The wind mechanical power $\dot{E}_{\mathrm{K}, \mathrm{X}}=(1 / 2) \dot{M}_{\text {out } \mathrm{X}} v_{\text {out }, \mathrm{X}}{ }^{2} \approx 2 \times 10^{45} \mathrm{erg} \mathrm{s}^{-1}$ corresponds to $\sim 15 \%$ of the AGN luminosity and is higher than the minimum value of $\sim 0.5 \%$ required for AGN feedback ${ }^{24}$ (see Supplementary Information). Therefore, this wind will probably have a strong influence on the host galaxy environment. An alternative interpretation of the $E \approx 9 \mathrm{keV}$ absorption trough with an ionized Fe K edge and an associated relatively slow wind (see Extended Data Fig. 1) is statistically disfavoured (see Methods).

Likewise, the case whereby the Fe K profile arises from relativistically blurred X-ray reflection off the inner accretion disk is also ruled out (see Methods and Supplementary Information). Such a model fails to predict the hard X-ray emission above $10 \mathrm{keV}$ (Extended Data Fig. 1). This case is also inconsistent with the variability observed during the observation (see Methods and Extended Data Figs 2 and 3). The fast wind model provides a self-consistent explanation of the variability in terms of a change in the absorber column density (Extended Data Table 2). In contrast, within the relativistic reflection model, the resultant variability pattern is the opposite of that expected from relativistic light bending ${ }^{25}$, with a dramatic increase in reflection fraction from the low to the high flux state (see Extended Data Table 3). Finally, the extrapolated hard X-ray luminosity from the fast-wind model matches the value expected from mid-infrared emission line diagnostics, while the relativistic reflection model underestimates this value by more than one order of magnitude (see Supplementary Information).

Observations from the Herschel Space Observatory using the Photodetector Array Camera and Spectrometer (PACS) show a massive molecular outflow ${ }^{6}$ in the absorption profile of the $\mathrm{OH}$ doublet at wavelength $\lambda=119.23 \mu \mathrm{m}$ of IRAS F11119+3257 (Fig. 2). This galaxy also shows clear signatures of high-velocity outflows $\left(\sim 1,000 \mathrm{~km} \mathrm{~s}^{-1}\right)$ at other energies, including neutral ${ }^{26}$ and moderately ionized ${ }^{27,28}$ gas. From our radiative transfer model $^{7}$ of the Herschel observation of the $119.23 \mu \mathrm{m}$ $\mathrm{OH}$ absorption profile (see Methods and Fig. 2) we estimate an outflow of around $300 \mathrm{pc}$ with average velocity of $v_{\text {out }, \mathrm{OH}}=1,000 \pm 200 \mathrm{~km} \mathrm{~s}^{-1}$, covering factor $C_{\mathrm{F}, \mathrm{OH}}=0.20 \pm 0.05$ and mass outflow rate of $\dot{M}_{\mathrm{out}, \mathrm{OH}}=$ $800_{-550}^{+1,200} M_{\odot} \mathrm{yr}^{-1}$ (see Supplementary Information). The momentum flux of the molecular outflow is considerably larger than that of the AGN radiation, $\dot{P}_{\text {out }, \mathrm{OH}}=11.0_{-7.5}^{+14.1} \dot{P}_{\text {rad }}$. Moreover, the mechanical power

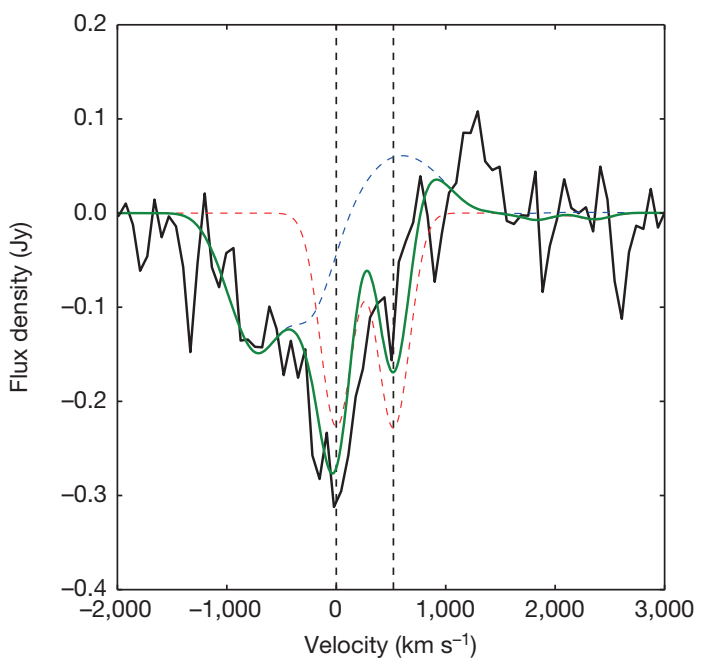

Figure $2 \mid$ Herschel-PACS OH $\lambda=119.23 \mu \mathrm{m}$ observation of IRAS F11119+3257. The blue (high velocity) and red (systemic velocity) dashed lines represent the different components of the outflow model. The green line represents the total best-fit model. The dashed vertical lines indicate the position of the $\mathrm{OH} \lambda=119.23 \mu \mathrm{m}$ doublet components relative to the blue line of the doublet $(\lambda=119.23 \mu \mathrm{m})$ and with respect to the systemic redshift of the galaxy. The average outflow velocity estimated from the $\mathrm{OH}$ model is $1,000 \pm 200 \mathrm{~km} \mathrm{~s}^{-1}$. corresponds to $\sim 2 \%$ of the AGN luminosity (see Supplementary Information), a value higher than the minimum required for quasar mode feedback ${ }^{24}$. The large velocity and energetics of this molecular outflow also favour an AGN origin ${ }^{3-5}$. These parameters are comparable to those found in similar AGN-dominated ultraluminous infrared galaxies ${ }^{3-8}$.

The two main mechanisms invoked to explain AGN feedback ${ }^{1,2}$ are the radio mode and the quasar or wind mode. In these cases the molecular outflows are accelerated by the interaction with either a powerful, but highly collimated, radio jet ${ }^{29}$ or a mildly relativistic accretion-disk wind $^{9-12}$, respectively. Powerful jets are found in radio-loud AGNs, but they constitute only a minority of the AGN population ${ }^{29}$. IRAS F11119+ 3257 , and ultraluminous infrared galaxies in general, do not show clear evidence for powerful jets ${ }^{6,8,30}$. Therefore, the radio-mode feedback is strongly disfavoured in this case.

Theoretical models of quasar-mode AGN feedback ${ }^{9-12,22}$ indicate that the shock caused by the interaction between the putative mildly relativistic AGN wind and the interstellar medium divides the resultant largescale outflow into two regimes. Momentum-conserving flows occur if the shocked wind gas can cool and most of the kinetic energy is radiated away. On the other hand, energy-conserving flows occur if the shocked wind gas is not efficiently cooled, and instead expands as a hot bubble. Conservation of energy leads to the relation ${ }^{9,10} \dot{P}_{\text {out }}=f\left(v_{\text {in }} /\right.$ $\left.v_{\text {out }}\right)\left(L_{\mathrm{AGN}} / c\right)$ where $v_{\text {in }}$ is the velocity of the inner X-ray wind, $v_{\text {out }}$ the velocity of the molecular outflow and $f=\mathrm{C}_{\mathrm{F} \text {,out }} / \mathrm{C}_{\mathrm{F} \text {,in }}$ is the ratio between the covering fractions of the outer molecular outflow and inner disk wind. This parameter $f$ indicates the efficiency or fraction of inner wind power that is transferred to the large-scale outflow. Substituting the values from the observed X-ray and $\mathrm{OH}$ winds in IRAS F11119+ 3257 we derive an efficiency of $f=0.22 \pm 0.07$.

In Fig. 3 we show the momentum fluxes of the inner X-ray wind and the molecular outflow observed in IRAS F11119+3257 with respect to their velocities, normalized to the momentum flux of the AGN radiation. For comparison, we consider also the energetics of mildly relativistic $\mathrm{X}$-ray winds detected in quasars ${ }^{15-19}$ and the molecular outflows detected

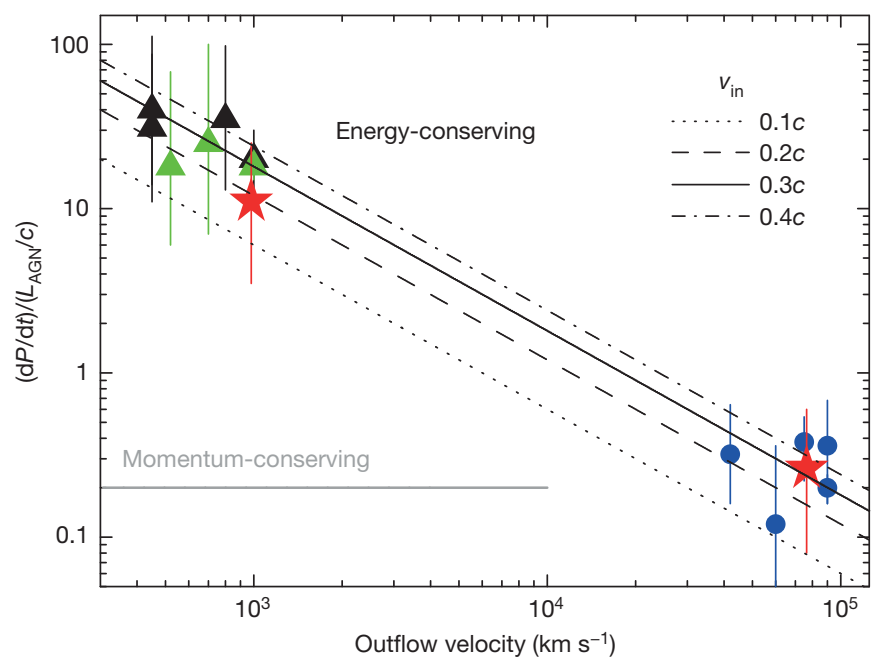

Figure $3 \mid$ Comparison between the inner winds and the molecular outflows. The momentum flux $(\mathrm{d} P / \mathrm{d} t)$ normalized to the radiation $\left(L_{\mathrm{AGN}} / c\right)$ is plotted against the wind outflow velocity. $v_{\text {in }}$ is the velocity of the inner X-ray wind, given in multiples of $c$. The disk and molecular winds in IRAS F11119+3257 (red filled stars), the disk winds of other quasars ${ }^{15-19}$ (blue filled circles) and the molecular outflows of other ultraluminous infrared galaxies $\left(\mathrm{OH}^{5,7}\right.$ green and $\mathrm{CO}^{4,8}$ black filled triangles) are reported. Uncertainties are $1 \sigma$. The dotted, dashed, solid and dot-dashed black curves represent the energy-conserving trends ${ }^{9-11}$ for different $v_{\text {in }}$ values. The horizontal grey line indicates the momentum-conserving case. An efficiency of $f=0.2$ is assumed. The momentum rates of the disk winds are multiplied by $f$. 
in ultraluminous infrared galaxies ${ }^{3-8}$ with similar AGN luminosities (see Supplementary Information and Extended Data Table 4). We consider the derived efficiency of $f=0.2$. Using the relation for an energy-conserving flow ${ }^{9-11}$ we find that the energetics of the molecular outflows would require initial AGN winds with a range of velocities between $v_{\text {in }} \approx(0.1-0.4) c$ (Fig. 3$)$. Indeed, substituting the estimated values for IRAS F11119+3257 of $v_{\text {in }} \approx 0.2 c, v_{\text {out }} \approx 1,000 \mathrm{~km} \mathrm{~s}^{-1}$ and $f \approx 0.2$ we obtain a value of $\dot{P}_{\text {out }} \approx 12 L_{\mathrm{AGN}} / c$ that is consistent with the $\mathrm{OH}$ observation.

This would not be possible in the momentum-conserving case. In fact, considering that a fraction $f=0.2$ of the initial wind momentum rate is transferred to the host and taking into account the uncertainties, the maximum value for the inner-disk wind is $f \dot{P}_{\text {out, } \mathrm{X}}=0.6 L_{\mathrm{AGN}} / \mathrm{c}$. This is much smaller than the minimum value for the molecular outflow of $\dot{P}_{\text {out }, \mathrm{OH}}=3.5 L_{\mathrm{AGN}} / c$ (see Supplementary Information and Fig. 3).

The mass outflow rate of radiation-driven winds ${ }^{9,22}$ is $\dot{M}_{\text {in }} \approx \dot{M}_{\mathrm{Edd}}$, where $\dot{M}_{\mathrm{Edd}}=L_{\mathrm{Edd}} / \eta c^{2}$ is the Eddington accretion rate and $\eta$ is the accretion efficiency. Moreover, their momentum rate is comparable ${ }^{9,22,23}$ to that of the AGN radiation, $\dot{P}_{\text {in }} \approx \dot{M}_{\text {in }} v_{\text {in }} \approx \mathrm{L}_{\mathrm{Edd}} / c$. Therefore, the observed fast-wind velocity suggests ${ }^{9}$ a high accretion efficiency $\eta \approx v_{\text {in }} /$ $c \approx 0.25$. For a stellar velocity dispersion of $\sigma \approx 100 \mathrm{~km} \mathrm{~s}^{-1}$ from the $M_{\mathrm{BH}}-\sigma$ relation $^{9,11}$, theoretical models of energy-conserving outflows ${ }^{9}$ suggest a molecular outflow velocity of $v_{\text {out }} \approx 1,000 \mathrm{~km} \mathrm{~s}^{-1}$, a mass outflow rate of $\dot{M}_{\text {out }} \approx 1,000 M_{\odot} \mathrm{yr}^{-1}$ and a momentum rate $\dot{P}_{\text {out }} \approx(10-$ 20) $\dot{P}_{\text {rad }}$, in agreement with the observations. Moreover, the mechanical energy of the fast wind of $\sim 15 \% L_{\mathrm{AGN}}$ is consistent with the theoretical value $^{9}$ of $(\eta / 2) L_{\mathrm{AGN}} \approx 13 \% L_{\mathrm{AGN}}$. The observed power of the molecular outflow of $\sim 2 \% \mathrm{~L}_{\mathrm{AGN}}$ is also consistent with that of the fast wind with an efficiency $f \approx 0.2$. With the limitation that the fast $\mathrm{X}$-ray wind is observed now, while the large-scale molecular outflow is probably an integrated effect of such winds over a much longer period of time, there is a very good quantitative agreement between observations and theoretical models $^{9-11,22}$.

This supports the idea that AGN winds can indeed provide an efficient way to transfer energy to the interstellar medium with a high degree of isotropy ${ }^{12}$, as required by the existence of large-scale outflows in most ultraluminous infrared galaxies ${ }^{3-8}$. Moreover, these results are consistent with the evolutionary scheme $e^{6}$ of ultraluminous infrared galaxies in which AGN-driven winds can clear out the obscuring material from the central regions of the galaxy ${ }^{1,2}$, eventually uncovering the underlying quasar.

Online Content Methods, along with any additional Extended Data display items and Source Data, are available in the online version of the paper; references unique to these sections appear only in the online paper.

\section{Received 4 November 2014; accepted 26 January 2015.}

1. Fabian, A. Observational evidence of active galactic nuclei feedback. Annu. Rev. Astron. Astrophys. 50, 455-489 (2012).

2. Veilleux, S., Cecil, G. \& Bland-Hawthorn, J. Galactic winds. Annu. Rev. Astron. Astrophys. 43, 769-826 (2005).

3. Fischer, J. et al. Herschel-PACS spectroscopic diagnostics of local ULIRGs: conditions and kinematics in Markarian 231. Astron. Astrophys. 518, L41 (2010).

4. Feruglio, C. et al. Quasar feedback revealed by giant molecular outflows. Astron. Astrophys. 518, L155 (2010).

5. Sturm, E. et al. Massive molecular outflows and negative feedback in ULIRGs observed by Herschel-PACS. Astrophys. J. 733, L16 (2011).

6. Veilleux, S. etal. Fast molecular outflows in luminous galaxy mergers: evidence for quasar feedback from Herschel. Astrophys. J. 776, 27 (2013).

7. González-Alfonso, E. et al. The Mrk 231 molecular outflow as seen in $\mathrm{OH}$. Astron. Astrophys. 561, A27 (2014)

8. Cicone, C. et al. Massive molecular outflows and evidence for AGN feedback from CO observations. Astron. Astrophys. 562, A21 (2014).
9. Zubovas, K. \& King, A. Clearing out a galaxy. Astrophys. J. 745, L34 (2012).

10. Faucher-Giguère, C.-A. \& Quataert, E. The physics of galactic winds driven by active galactic nuclei. Mon. Not. R. Astron. Soc. 425, 605-622 (2012).

11. Zubovas, K. \& Nayakshin, S. Energy- and momentum-conserving AGN feedback outflows. Mon. Not. R. Astron. Soc. 440, 2625-2635 (2014).

12. Wagner, A. Y., Umemura, M. \& Bicknell, G. V. Ultrafast outflows: galaxy-scale active galactic nucleus feedback. Astrophys. J. 763, L18 (2013).

13. Tombesi, F. et al. Evidence for ultra-fast outflows in radio-quiet AGNs. I. Detection and statistical incidence of Fe K-shell absorption lines. Astron. Astrophys. 521, A57 (2010).

14. Gofford, J. et al. The Suzaku view of highly ionized outflows in AGN.-I. Statistical detection and global absorber properties. Mon. Not. R. Astron. Soc. 430, 60-80 (2013).

15. Chartas, G., Saez, C., Brandt, W. N., Giustini, M. \& Garmire, G. P. Confirmation of and variable energy injection by a near-relativistic outflow in APM 08279+5255. Astrophys. J. 706, 644-656 (2009).

16. Pounds, K. A. \& Reeves, J. N. Quantifying the fast outflow in the luminous Seyfert galaxy PG1211+143. Mon. Not. R. Astron. Soc. 397, 249-257 (2009).

17. Lanzuisi, G. et al. HS 1700+6416: the first high-redshift unlensed narrow absorption line-QSO showing variable high-velocity outflows. Astron. Astrophys. 544, A2 (2012).

18. Chartas, G. et al. Magnified views of the ultrafast outflow of the $z=1.51$ active galactic nucleus HS 0810+2554. Astrophys. J. 783, 57 (2014).

19. Gofford, J. et al. Revealing the location and structure of the accretion disk wind in PDS 456. Astrophys. J. 784, 77 (2014).

20. Kawakatu, N., Imanishi, M. \& Nagao, T. Anticorrelation between the mass of a supermassive black hole and the mass accretion rate in type 1 ultraluminous infrared galaxies and nearby QSOs. Astrophys. J. 661, 660-671 (2007).

21. Teng, S. H. \& Veilleux, S. X-QUEST: a comprehensive $x$-ray study of local ULIRGS and QSOs. Astrophys. J. 725, 1848-1876 (2010).

22. King, A. R. \& Pounds, K. A. Black hole winds. Mon. Not. R. Astron. Soc. 345, 657-659 (2003).

23. Reynolds, C. S. Constraints on Compton-thick winds from black hole accretion disks: can we see the inner disk? Astrophys. J. 759, L15 (2012).

24. Hopkins, P. F. \& Elvis, M. Quasar feedback: more bang for your buck. Mon. Not. R. Astron. Soc. 401, 7-14 (2010).

25. Miniutti, G. \& Fabian, A. C. A light bending model for the X-ray temporal and spectral properties of accreting black holes. Mon. Not. R. Astron. Soc. 349, $1435-1448$ (2004).

26. Rupke, D. S., Veilleux, S. \& Sanders, D. B. Outflows in active galactic nucleus/ starburst-composite ultraluminous infrared galaxies. Astrophys. J. 632, 751-780 (2005)

27. Lipari, S. et al. Extreme galactic wind and Wolf-Rayet features in infrared mergers and infrared quasi-stellar objects. Mon. Not. R. Astron. Soc. 340, 289-303 (2003)

28. Spoon, H. W. W. \& Holt, J. Discovery of strongly blueshifted mid-infrared [Ne III] and [Ne V] emission in ULIRGs. Astrophys. J. 702, L42-L46 (2009).

29. Tadhunter, C., Morganti, R., Rose, M., Oonk, J. B. R. \& Oosterloo, T. Jet acceleration of the fast molecular outflows in the Seyfert galaxy IC 5063. Nature 511, 440-443 (2014)

30. Nagar, N. M., Wilson, A. S., Falcke, H., Veilleux, S. \& Maiolino, R. The AGN content of ultraluminous IR galaxies: high resolution VLA imaging of the IRAS 1 Jy ULIRG sample. Astron. Astrophys. 409, 115-121 (2003).

Supplementary Information is available in the online version of the paper.

Acknowledgements F.T. would like to thank T. Kallman, J. García, F. Tazaki, F. Paerels and M. Cappi for comments. F.T. acknowledges support from NASA (grant NNX12AH4OG). M.M. and S.V. are supported in part by NASA grants NHSC/JPL RSA 1427277 and 1454738. S.V. also acknowledges partial support through grant NSF-AST1009583. J.N.R. acknowledges the financial support of the STFC. E.G.-A. is a Research Associate at the Harvard-Smithsonian Center for Astrophysics, and thanks the Spanish Ministerio de Economía y Competitividad for support under projects AYA2010-21697-C05-0 and FIS2012-39162-C06-01. C.S.R. thanks support from NASA (grant NNX14AF86G) and the US National Science Foundation (grant AST1333514)

Author Contributions F.T. is the Principal Investigator of the Suzaku observation. He led the X-ray spectral analysis, interpretation of the results and manuscript preparation. M.M. and E. G.-A. performed the analysis and modeling of the Herschel data. S.V. contributed to the interpretation of the results. J.N.R. and C.S.R. contributed to the X-ray spectral analysis and interpretation of the results. All authors participated in the review of the manuscript.

Author Information Reprints and permissions information is available at www.nature.com/reprints. The authors declare no competing financial interests. Readers are welcome to comment on the online version of the paper. Correspondence and requests for materials should be addressed to F.T. (ftombesi@astro.umd.edu). 


\section{METHODS}

Suzaku observation log. IRAS F11119+3257 was observed with Suzaku for the period 13-19 May 2013 for a total exposure of $250 \mathrm{ks}$. The data reduction and analysis were performed following the standard procedures as described in the Suzaku data reduction guide. We use the heasoft version 6.12 package and the latest calibration files (http://heasarc.nasa.gov/lheasoft/).

Suzaku XIS data reduction. We derived the Suzaku XIS cleaned event files and applied standard screening criteria. The $3 \times 3$ and $5 \times 5$ editing modes were combined. The source spectra were extracted from circular regions of $2.5^{\prime \prime}$ radius centred on the source. The background spectra were extracted from annular regions with inner and outer radii of $3-4^{\prime \prime}$ centred on the source and excluding contamination from the calibration sources. The spectra from the two front illuminated detectors, $\mathrm{XIS} 0$ and XIS3, were combined after verifying that the data are consistent with each other. Hereafter we refer to them as XIS03. The data of the back illuminated XIS1 detector are used as well. The XIS response and ancillary files were produced. The source count-rates (in units of counts per second) in the $E=0.5-10 \mathrm{keV}$ band are $0.0576,0.0649$ and 0.06 for the XIS0, XIS1 and XIS3 detectors, respectively. The background count-rates are $18 \%$ and $30 \%$ of the source count-rates for the XIS03 and XIS1 detectors, respectively.

Suzaku PIN data reduction. The Suzaku PIN source spectrum was extracted within the good time intervals (those during which events are accumulated) and corrected for the detector dead time. The latest response file was used. We use the latest and most accurate tuned non-X-ray background event file version 2.2 ver 1403 provided by the Suzaku team. The non-X-ray background event file was used as input to the hxdpinxbpi task and combined with a model of the cosmic X-ray background provided by the Suzaku team. We conservatively include a systematic uncertainty on the PIN background of $1.5 \%$. The source is detected in the PIN at the $4 \sigma$ level. The source count-rate in the $E=15-25 \mathrm{keV}$ band is $0.005 \pm 0.001$ counts s $^{-1}$, including the systematic error on the background. The average source count-rate is $3.3 \%$ of the background. There are no other X-ray sources with $2-10 \mathrm{keV}$ flux higher than $5 \times 10^{-14} \mathrm{erg} \mathrm{s}^{-1} \mathrm{~cm}^{-2}$ in the Chandra, XMM-Newton, BeppoSAX and ASCA archives, excluding contamination from other sources within the PIN field of view. Above $10 \mathrm{keV}$, we do not find any source detected within a $1^{\circ}$ radius in the Swift BAT 70-month, RXTE and Integral surveys. The PIN flux of IRAS F1111+3257 is also below the sensitivity limit of these hard X-ray surveys.

Suzaku spectral analysis. The spectral analysis was carried out using the software XSPEC version 12.7.1 (http://heasarc.gsfc.nasa.gov/xanadu/xspec/). All uncertainties quoted are at the $1 \sigma$ level for one parameter of interest and the energies are reported in the source rest-frame, unless otherwise stated. We exploit the broadband capabilities of Suzaku, combining the $E=0.5-10.5 \mathrm{keV}$ XIS and $E=15-$ $25 \mathrm{keV}$ PIN spectra. We perform joint fits of the Suzaku XIS03 and XIS1 spectra excluding the energy range around the Si K edge $(E=1.5-2 \mathrm{keV})$, which is known to be affected by calibration issues. The XIS03/XIS1 cross-normalization was left free to vary, but it was always found to be consistent within $3 \%$. We take into account the XIS/PIN cross-normalization of $1.16 \pm 0.01$. All spectra were grouped to a minimum of 25 counts per energy bin in order to allow the use of the $\chi^{2}$ minimization in the model fitting. Throughout we include a neutral Galactic absorption of $N_{\mathrm{H}}=$ $2.1 \times 10^{20} \mathrm{~cm}^{-2}$.

Fast-wind model. We model the broad absorption at $E \approx 9 \mathrm{keV}$ with the $X S T A R^{31}$ code version $2.2 .1 \mathrm{bn}$. We consider a $\Gamma=2$ power-law continuum, consistent with the observed value (see Supplementary Information), and standard solar abundances. A turbulent velocity of $30,000 \mathrm{~km} \mathrm{~s}^{-1}$ is assumed for the fast-wind model. This high value is introduced only to model the large width of the absorption line and it is probably not linked to an actual physical turbulence in the gas. For instance, detailed accretion-disk wind models show that the line profiles become much broader because of the velocity shear between consecutive zones of the wind ${ }^{32}$.

This provides a very good fit to the data, with $\gamma^{2} / v=1410.4 / 1391$, providing a higher fit improvement, comparable to the phenomenological best-fit case of a broad absorption line $\left(\Delta \chi^{2} / \Delta v=54.2 / 3\right)$. This corresponds to a very high detection confidence level of $6.5 \sigma$. The relative column density, ionization and outflow velocity are $N_{\mathrm{H}}=6.4_{-1.3}^{+0.8} \times 10^{24} \mathrm{~cm}^{-2}, \log \xi=4.11_{-0.04}^{+0.09} \mathrm{erg} \mathrm{s}^{-1} \mathrm{~cm}$ and $v_{\mathrm{out}, \mathrm{X}}=0.255 \pm$ $0.011 c$, respectively (see Extended Data Table 1). The absorber is consistent with fully covering the source, with a lower limit of the covering fraction of $C_{\mathrm{F}, \mathrm{X}}>0.85$ at the $90 \%$ significance level (see Supplementary Information). The fast-wind model is able to simultaneously take into account both the broad absorption at $E \approx 7-$ $10 \mathrm{keV}$ and the excess of flux at $E=15-25 \mathrm{keV}$ (see Extended Data Fig. 1c).

We note that a more physical model of a high-column-density wind should include both Compton scattering and emission (for the latter see Supplementary Information). A few such models ${ }^{33,34}$ have been recently reported, but they are not publicly available for use in XSPEC and require a relative fine-tuning of the parameters. Moreover, the wind parameters are well approximated with XSTAR absorption tables ${ }^{33,34}$, supporting our conclusions. The effects of Compton scattering should be marginal, introducing a continuum break at an energy beyond the observed
Suzaku bandpass of $E \approx 50 \mathrm{keV}$ and contribute to the broadening of the lines of less than $\sim 0.3 \mathrm{keV}$.

Slow-wind model. Considering the slow-wind model, in which the feature at $E \approx$ $9 \mathrm{keV}$ is identified with an ionized $\mathrm{Fe} \mathrm{K}$ absorption edge, the data require a lower $X S T A R$ turbulent velocity width of $500 \mathrm{~km} \mathrm{~s}^{-1}$. This provides an overall good fit of the data, with $\chi^{2} / v=1,439.3 / 1,391$. The relative column density, ionization and outflow velocity are $N_{\mathrm{H}}=5.5_{-0.7}^{+0.6} \times 10^{24} \mathrm{~cm}^{-2}, \log \xi=3.77_{-0.07}^{+0.08} \mathrm{erg} \mathrm{s}^{-1} \mathrm{~cm}$ and $v_{\text {out }}=$ $0.0258_{-0.0128}^{+0.0109} c$, respectively. The lower limit of the covering fraction is $C_{\mathrm{F}}>0.93$ at the $90 \%$ significance level. Even though it provides an overall sufficient representation of the data, this fit is statistically much worse than the previous case of a fast wind (see Extended Data Fig. 1d). In fact, the $\chi^{2}$ difference for the same number of degrees of freedom with respect to the fast-wind model is $\Delta \chi^{2}=28$.9. In particular, this slow-wind model does not provide a good fit for the absorption trough at $E \approx$ $7-10 \mathrm{keV}$.

Relativistic reflection model. We use the most accurate relativistic reflection code available in the literature, the "relxill p" model ${ }^{35}$. This model considers the lamp post geometry in which the compact X-ray source is located at a certain height along the rotation axis of the black hole in units of gravitational radii $r_{\mathrm{g}}=G M_{\mathrm{BH}} / c^{2}$. The reflection fraction and emissivity index are self-consistently calculated by the model depending on the source height and black hole spin. We consider a typical outer-disk radius of $r_{\text {out }}=400 r_{\mathrm{g}}$ and the inner radius $r_{\text {in }}$ is linked to the innermost stable circular orbit for a given black-hole spin value. We consider standard solar abundances.

The free parameters are the height of the illuminating source $h$, the disk inclination $i$, the ionization parameter $\log \xi$, the normalization and the black-hole spin $a$. This model provides a fit statistics of $\chi^{2} / v=1413.2 / 1390$. The best-fit parameters suggest a source height of just $h=2.2_{-0.6}^{+1.3} r_{\mathrm{g}}\left(r_{\mathrm{g}}=G M_{\mathrm{BH}} / c^{2}\right)$ and a rapidly spinning black hole with $a>0.85$. The material is mildly ionized, with $\log \xi=3.15_{-0.09}^{+0.15} \mathrm{erg} \mathrm{s}^{-1}$ $\mathrm{cm}$, and the disk inclination is estimated to be $i=52_{-6}^{+4}$ degrees. Letting the reflection parameter vary freely we find a very high best-fit value of $R \approx 6$. The relativistic reflection model provides a relatively good representation of the data up to $E \approx 10 \mathrm{keV}$ but it is not able to model the excess of flux at $E=15-25 \mathrm{keV}$ (see Extended Data Fig. 1e).

Time-resolved spectral analysis. The source lightcurve shows a factor-of- 10 variability in the $4-10 \mathrm{keV}$ count-rate over an interval of $\sim 3$ days between the minimum and maximum values (Extended Data Fig. 2). This variability must be driven by the change of some parameters, such as the continuum, absorption or reflection. We performed a time-resolved spectral analysis by splitting the observation at $377 \mathrm{ks}$ after the beginning (Extended Data Fig. 2). This allows us to have the same signalto-noise ratio in the $E=4-10 \mathrm{keV}$ band for the low-flux (first) and high-flux (second) intervals. The source shows a much smaller variability of a factor of $\sim 1.5$ in the PIN data at $E=15-25 \mathrm{keV}$. The broad-band spectra extracted in the lowflux and high-flux intervals are shown in Extended Data Fig. 3a.

We performed a combined fit of the low-flux and high-flux intervals using alternately the fast-wind model and the relativistic-reflection model and compared the values of the parameters to determine the main driver of the variability in these two cases.

Regarding the fit with the fast-wind model, we test three different variability cases: (1) constant fully covering absorption with variable power-law continuum; (2) constant power-law continuum with variable partial covering absorption; (3) constant power-law continuum and variable fully covering absorption. Cases (1) and (3) provide the best representations of the data with comparable statistics of $\chi^{2} / v=$ $1,757.9 / 1,692$ and $\chi^{2} / v=1,757.3 / 1,693$, respectively. In case (1) of the constant absorber and variable continuum we find that the main change in the fit parameters may be due to an increase in the normalization of the continuum with $\Gamma \approx 2$. The continuum slope is consistent in the two intervals. The relative ionizing luminosity between 1 Ryd and 1,000 Ryd (where $1 \mathrm{Ryd}=13.6 \mathrm{eV}$ ) would be increasing from $\log L_{\text {ion }} \approx 45.2 \mathrm{erg} \mathrm{s}^{-1}$ to $\log L_{\text {ion }} \approx 45.7 \mathrm{erg} \mathrm{s}^{-1}$. Given that the column density and velocity of the absorber are constant, this increase in luminosity by a factor of $\sim 3.4$ should have caused an increase in ionization by the same factor, fully ionizing the gas, to the point that it would have not been detected in the second interval. This consideration alone strongly disfavours the continuum-variability case. In case (3) of a constant continuum and variable absorber we find that the main driver of the flux variability is a $40 \%$ decrease in column density from $N_{\mathrm{H}} \approx 8.1 \times 10^{24} \mathrm{~cm}^{-2}$ to $N_{\mathrm{H}} \approx 4.9 \times 10^{24} \mathrm{~cm}^{-2}$ between the two intervals (Extended Data Table 2). Moreover, the fact that the observed change in flux of a factor of $\sim 10$ is much larger at $E=4-10 \mathrm{keV}$ compared to a factor of only $\sim 1.5$ at $E>15 \mathrm{keV}$ also points to the fact that the main variability is due to a change in absorbing column density rather than continuum emission with constant slope (see Extended Data Fig. 3b).

Regarding the fit with the relativistic-reflection model, we tested two different variability cases: (1) constant reflection with variable power-law continuum and (2) constant power-law continuum with variable reflection (that is, source height and ionization). Case (1) would indicate a constant and very small source height of $h<$ $2 r_{\mathrm{g}}$. Instead, the continuum slope would be required to change from the unrealistically 
high value of $\Gamma \approx 2.7$ to $\Gamma \approx 2.2$ between the two intervals. Overall, this case does not provide a good fit to the data, with a poor statistics of $\chi^{2} / v=1,878.7 / 1,692$. Case (2) provides a better fit of $\chi^{2} / v=1,766.4 / 1,693$. The fit with the relativistic reflection model provides a relatively good representation of the data up to $E \approx$ $10 \mathrm{keV}$ but not at higher energies (see Extended Data Fig. 3b). In this case, the direct continuum is required to be constant between the two intervals and the main driver of the variability would be the height of the X-ray source, decreasing from $h=5.4_{-1.2}^{+54.4} r_{\mathrm{g}}$ down to $h<3.2 r_{\mathrm{g}}$ from the low-flux to the high-flux case, respectively (Extended Data Table 3 ). This corresponds to an increase in reflection fraction from $\sim 3$ in the low-flux interval up to the maximum value allowed of $\sim 10$ for the high-flux case. Even though this fit might provide a relatively good representation of the data, it clearly contradicts the variability expected from the associated relativistic light bending case ${ }^{25}$, which predicts a very specific variability pattern of higher/lower reflection fractions (lower/higher X-ray source heights) for lower/ higher fluxes.

Suzaku XIS background and systematics check. We investigated the existence of the broad absorption at $E \approx 9 \mathrm{keV}$, also performing independent fits of the Suzaku XIS0, XIS1 and XIS3 detectors. In all cases the parameters are consistent at the $90 \%$ level and in particular the broad absorption is independently detected at the $3 \sigma$ level in the XIS0 and XIS1 and at the $4 \sigma$ level for the XIS3 (Fig. 1). The XIS cameras have an instrumental background emission line from $\mathrm{Ni} \mathrm{K} \alpha$ at the observed energy of $7.47 \mathrm{keV}$. The line intensity is slightly dependent on the location on the detector. Therefore, an incorrect background selection might possibly induce spurious absorption/emission lines in the background-subtracted spectrum. We performed several tests in order to exclude this possibility. First, we note that the observed centroid energy of the broad line of $E=8.26_{-0.34}^{+0.64} \mathrm{keV}$ is not consistent with the energy of the instrumental line. Second, the background emission line is unresolved, with a width of $\sigma_{E}<100 \mathrm{eV}$. This is not consistent with the large width of the absorption feature of $\sigma_{E} \approx 1 \mathrm{keV}$. Third, we performed fits using background regions extracted from one, two and three circles of $2.5^{\prime \prime}$ size away from the source and annuli with inner/outer radii of $3^{\prime \prime} / 5^{\prime \prime}$ and $4^{\prime \prime} / 5^{\prime \prime}$ centred on the source. Fourth, we performed an additional consistency check considering a smaller circular source extraction region of $1.5^{\prime \prime}$ and an annular background region of the same area with inner/outer radii of $3 " / 3.4^{\prime \prime}$. This allows us to derive a cleaner extraction region, with a marginal loss of $20 \%$ of the source counts in the $E=4-10 \mathrm{keV}$ band, and to drastically minimize the background, reducing its count-rate by a factor of 2.5. In all cases we find that the best-fit parameters, and in particular those of the broad absorption feature, are always consistent within the $1 \sigma$ errors. Considering only the XIS data and fitting both the fast- and slow-wind models and the relativistic reflection model we obtain parameters that are consistent within the $1 \sigma$ errors with those derived using the combined XIS and PIN data. This indicates that these models depend only weakly on the $E=15-25 \mathrm{keV}$ PIN data. The fast-wind $\left(\chi^{2} / v=1,372.1 / 1,365\right)$ and the relativistic reflection $\left(\chi^{2} / v=1,369.2 / 1,364\right)$ models provide statistically comparable fits of the XIS data alone. Instead, the fit using the slow-wind model is statistically worse $\left(\chi^{2} / v=1,404.6 / 1,365\right)$.

Modelling of the Herschel $\mathrm{OH}$ spectrum. The profile of the $\mathrm{OH} 119.23 \mu \mathrm{m}$ doublet shows ${ }^{6}$ a strong asymmetry at systemic velocities and an obvious blue wing extending well beyond $1,000 \mathrm{~km} \mathrm{~s}^{-1}$ (see Fig. 2). This profile is modelled in spherical symmetry as an extended outflow on the scale of $300 \mathrm{pc}$. The Spitzer space telescope's Infrared Spectrograph (IRS) low-resolution spectra, the Infrared Astronomical Satellite (IRAS) fluxes, and Herschel spectrum around the $\mathrm{OH}$ doublet are used to estimate the underlying continuum.

The covering factor of the outflow is estimated to be $C_{\mathrm{F}, \mathrm{OH}}=0.20 \pm 0.05$. The sum of the covering factors of the outflow and systemic components is close to unity, consistent with the fraction of gas affected/unaffected by the outflow. The outflow velocity is also well determined, with an average value of $v_{\text {out,OH }}=1,000 \pm 200 \mathrm{~km} \mathrm{~s}^{-1}$. The absorption is seen in front of the far-infrared continuum, which is well modelled with a dust radius of $R_{\text {dust }}=250 \mathrm{pc}$. The distance of the $\mathrm{OH}$ absorber is estimated at $r=300 \mathrm{pc}$ in radius (or $600 \mathrm{pc}$ in diameter). This value is consistent with previous results ${ }^{7}$ derived using the same model for the more detailed spectrum of the ultraluminous infrared galaxy Mrk231, indicating that the outflowing $\mathrm{OH}$ is mostly linked to the source of far-infrared continuum.

The hydrogen number density is derived from the equation $n_{\mathrm{H}}=N_{\mathrm{OH}} /\left(\mathrm{X}_{\mathrm{OH}} \times\right.$ $\Delta r$ ), where $N_{\mathrm{OH}}$ is the $\mathrm{OH}$ column density, $\mathrm{X}_{\mathrm{OH}}$ is the $\mathrm{OH}$ abundance relative to $\mathrm{H}$ and $\Delta r$ is the width of the region sampled by the outflowing $\mathrm{OH}$. The estimated value of the $\mathrm{OH}$ column density from the fit of the line profile is $N_{\mathrm{OH}}=3 \times$ $10^{16} \mathrm{~cm}^{-2}$. This value is conservatively low but enough to account for the optically thick $\mathrm{OH} 119.23 \mu \mathrm{m}$ absorption line. The standard value ${ }^{7}$ of the $\mathrm{OH}$ abundance relative to $\mathrm{H}$ in active regions where $\mathrm{OH}$ is highly abundant is $X_{\mathrm{OH}}=2 \times 10^{-6}$. The width of the outflowing $\mathrm{OH}$ region of $\Delta r=50 \mathrm{pc}$ is derived from the relation $R_{\text {out }}=1.2 R_{\text {dust }}$, which is based on more accurate models ${ }^{7}$ for the similar $\mathrm{OH}$ profile in Mrk 231. Therefore, we estimate a hydrogen number density of $n_{\mathrm{H}}=100 \mathrm{~cm}^{-3}$. Exploring the parameter space of the model, we find an uncertainty of a factor of two on this value (see Supplementary Information).

31. Kallman, T. \& Bautista, M. Photoionization and high-density gas. Astrophys. J. Suppl. Ser. 133, 221-253 (2001).

32. Fukumura, K. et al. Stratified magnetically driven accretion-disk winds and their relations to jets. Astrophys. J. 780, 120 (2014).

33. Sim, S. A., Miller, L., Long, K. S., Turner, T. J. \& Reeves, J. N. Multidimensional modelling of X-ray spectra for AGN accretion disc outflows-II. Mon. Not. $R$. Astron. Soc. 404, 1369-1384 (2010).

34. Hagino, K. et al. The origin of ultra-fast outflows in AGN: Monte-Carlo simulations of the wind in PDS 456. Mon. Not. R. Astron. Soc. 446, 663-676 (2015).

35. García, J. et al. Improved reflection models of black hole accretion disks: treating the angular distribution of X-rays. Astrophys. J. 782, 76 (2014). 

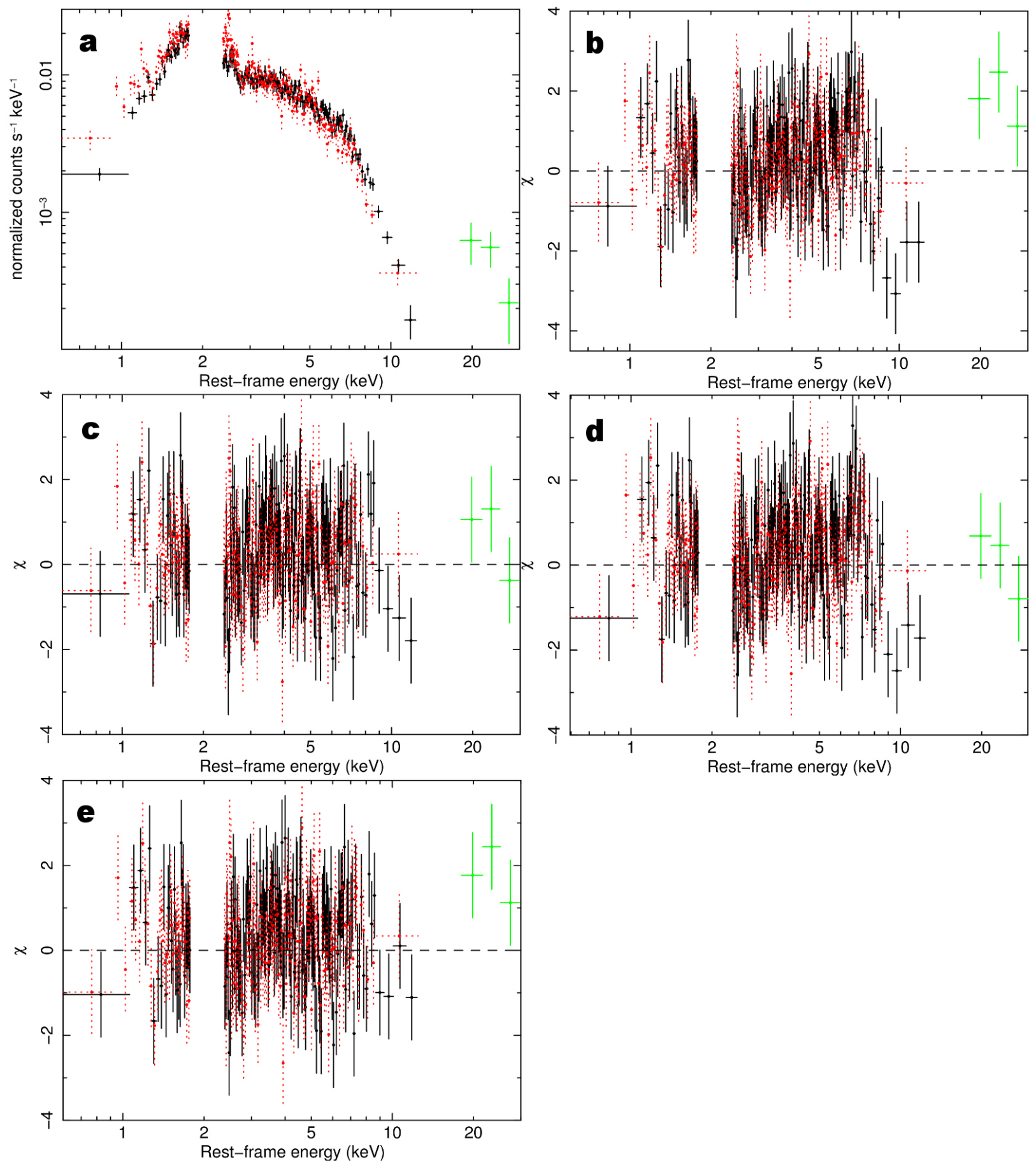

Extended Data Figure $1 \mid$ Broad-band Suzaku spectrum in the $E=0.5-$

power-law model, the fast-wind model, the slow-wind model and the $30 \mathrm{keV}$ band. a, The time-averaged Suzaku XIS03 (solid black), XIS1 (dotted red) and PIN (solid green) spectra binned to $10 \sigma, 5 \sigma$ and $3 \sigma$, respectively. The data-to-model residuals in units of sigma with respect to the absorbed relativistic reflection model are shown in $\mathbf{b}, \mathbf{c}, \mathbf{d}$ and $\mathbf{e}$, respectively. The energy is in the rest-frame and errors are at the $1 \sigma$ level. 


\section{RESEARCH LETTER}

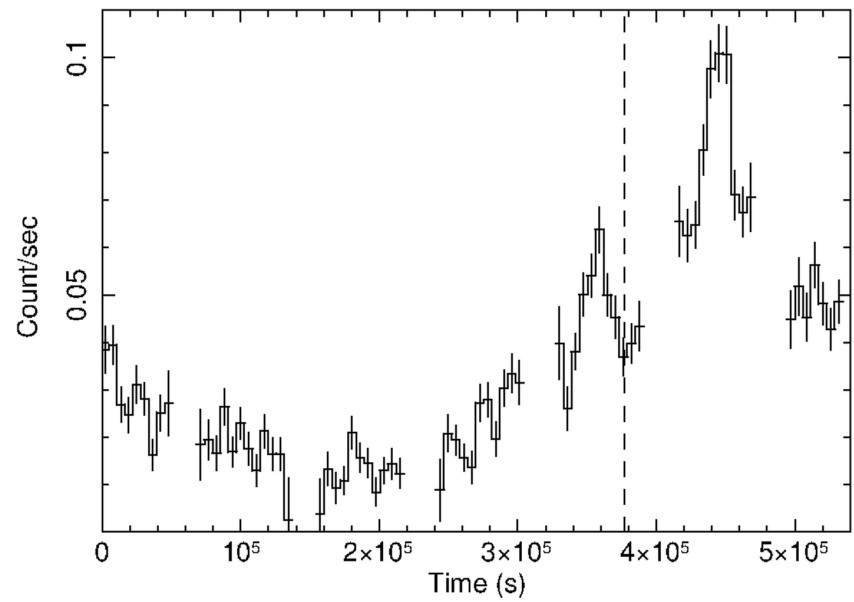

Extended Data Figure 2 Background-subtracted Suzaku XIS03 light curve in the $E=4-10 \mathrm{keV}$ band. The data are binned to the Suzaku orbital period of $5,760 \mathrm{~s}$. The vertical line indicates the time at which the observation is split into two parts for the time-resolved spectral analysis. The effective on-source exposure time is $250 \mathrm{ks}$. The gaps in the light curve indicate periods in which the satellite could not point to the source. Therefore, the total temporal coverage of the observation is longer, about $500 \mathrm{ks}$. Errors are at the $1 \sigma$ level. 


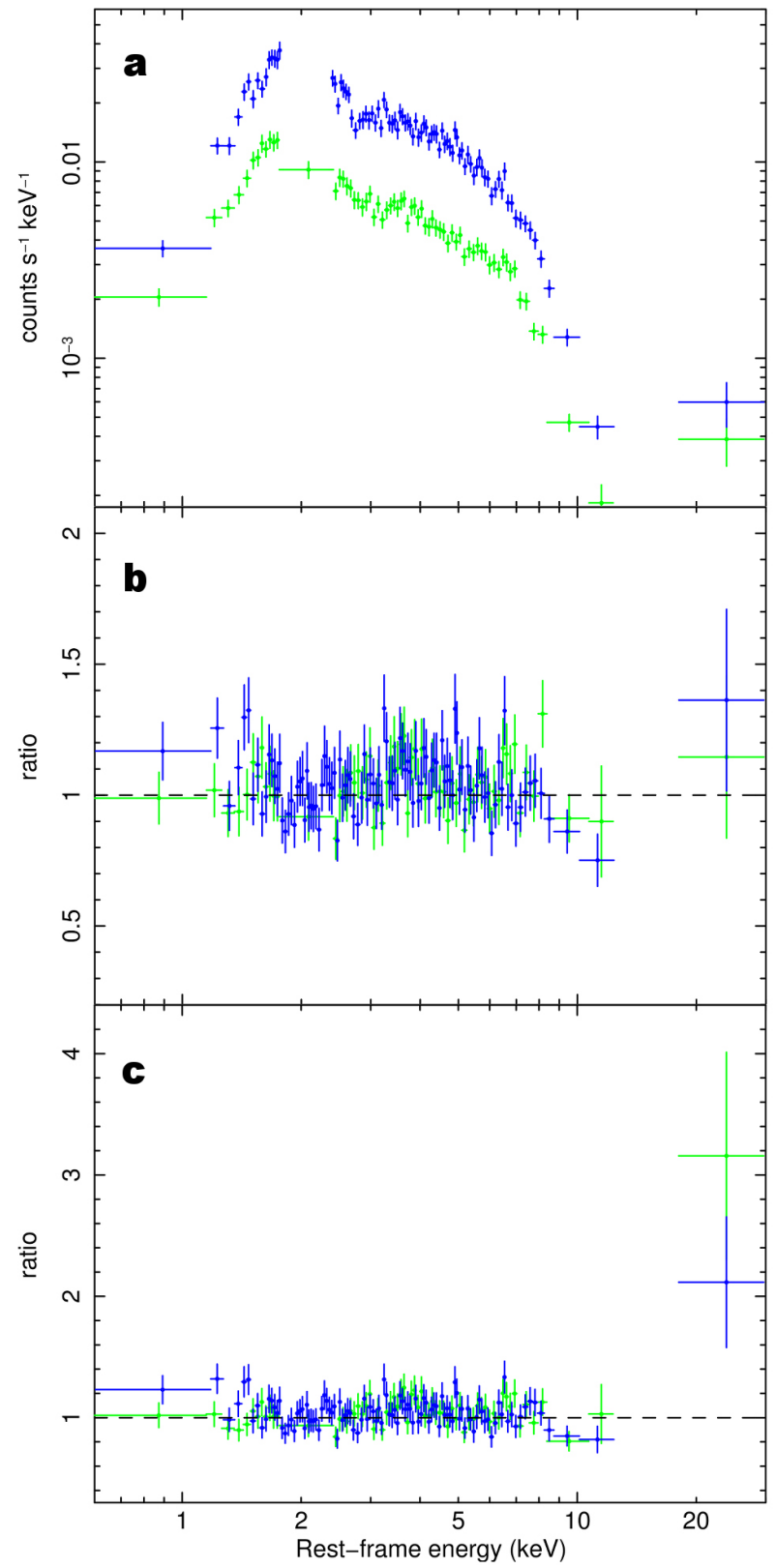

Extended Data Figure 3 Time-resolved Suzaku spectral analysis in the $\boldsymbol{E}=\mathbf{0 . 5}-30 \mathrm{keV}$ band. a, Suzaku XIS03 and PIN spectra extracted during the low-flux (green) and high-flux (blue) intervals. The XIS03 and PIN data are rebinned to $10 \sigma$ and $5 \sigma$, respectively. The data-to-model ratios with respect to the fast-wind model and relativistic-reflection model are reported in $\mathbf{b}$ and $\mathbf{c}$, respectively. Errors are at the $1 \sigma$ level. 


\section{RESEARCH LETTER}

Extended Data Table 1 | Best-fit values of the fast-wind model for the time-averaged spectrum

\begin{tabular}{|c|c|c|c|}
\hline \multicolumn{4}{|c|}{ Absorbed Power-law } \\
\hline г & $\begin{array}{c}N_{H} \\
\left(10^{22} \mathrm{~cm}^{-2}\right)\end{array}$ & & $\chi^{2} / \nu$ \\
\hline $2.04 \pm 0.03$ & $2.07 \pm 0.08$ & & $1410.4 / 1391$ \\
\hline \multicolumn{4}{|c|}{ Photoionized absorption } \\
\hline $\begin{array}{c}\log \xi \\
\left(\operatorname{erg~s}^{-1} \mathrm{~cm}\right)\end{array}$ & $\begin{array}{c}N_{H} \\
\left(10^{24} \mathrm{~cm}^{-2}\right)\end{array}$ & $\begin{array}{l}v_{\text {out }} \\
\text { (c) }\end{array}$ & $c_{F}$ \\
\hline $4.11_{-0.04}^{+0.09}$ & $6.4_{-1.3}^{+0.8}$ & $0.255 \pm 0.011$ & $>0.85$ \\
\hline
\end{tabular}

Errors are at the $1 \sigma$ level. 
Extended Data Table 2 | Best-fit values of the fast-wind model for the time-resolved spectral analysis

\begin{tabular}{|c|c|c|}
\hline & Low-Flux & High-Flux \\
\hline \multicolumn{3}{|l|}{ Absorbed power-law } \\
\hline $\begin{array}{l}\Gamma \\
N_{H}\left(10^{22} \mathrm{~cm}^{-2}\right)\end{array}$ & $\begin{array}{l}2.05 \pm 0.05 \\
2.06 \pm 0.12 \\
\end{array}$ & \\
\hline \multicolumn{3}{|c|}{ Photoionized absorption } \\
\hline $\begin{array}{l}\log \xi\left(\mathrm{erg} \mathrm{s}^{-1} \mathrm{~cm}\right) \\
N_{H}\left(10^{24} \mathrm{~cm}^{-2}\right) \\
v_{\text {out }}(\mathrm{c})\end{array}$ & $\begin{array}{c}4.08_{-0.03}^{+0.08} \\
8.1_{-1.1}^{+0.6} \\
0.243_{-0.021}^{+0.020} \\
\end{array}$ & $\begin{array}{c}4.13_{-0.08}^{+0.20} \\
4.9_{-1.9}^{+1.3} \\
0.268_{-0.034}^{+0.031} \\
\end{array}$ \\
\hline \multicolumn{3}{|l|}{ Best-fit statistics } \\
\hline$\chi^{2} / \nu$ & $1757.3 / 1692$ & \\
\hline
\end{tabular}

Errors are at the $90 \%$ level. 


\section{RESEARCH LETTER}

Extended Data Table 3 Best-fit values of the relativistic reflection model for the time-resolved spectral analysis

\begin{tabular}{lcc}
\hline & Low-Flux & High-Flux \\
\hline Absorbed power-law & & \\
\hline$\Gamma$ & $\begin{array}{c}2.01_{-0.04}^{+0.08} \\
2.24_{-0.10}^{+0.15}\end{array}$ \\
$N_{H}\left(10^{22} \mathrm{~cm}^{-2}\right)$ & & \\
\hline Relativistic reflection & & \\
\hline & & \\
$a$ & $>0.93$ & \\
$h\left(r_{g}\right)$ & $5.4_{-1.2}^{+54.4}$ & \\
$i($ degrees $)$ & $44_{-7}^{+5}$ & \\
log $\xi$ (erg s & \\
$R$ & $\left.3.05_{-0.07}^{+0.13} \mathrm{~cm}\right)$ & $3.32_{-0.09}^{+0.13}$ \\
\hline Best-fit statistics & $\simeq 3$ & $\simeq 10$ \\
\hline & & \\
$\chi^{2} / \nu$ & $1766.4 / 1693$ & \\
\hline
\end{tabular}

The parameters $a$ and $R$ refer to the black-hole spin and reflection fraction, respectively. Errors are at the $90 \%$ level. 
Extended Data Table 4 | Parameters of outflows in other quasars and ultraluminous infrared galaxies collected from the literature

\begin{tabular}{lcccc}
\hline \multicolumn{1}{c}{ Name } & $\begin{array}{c}\log L_{\text {AGN }} \\
\left(\mathrm{erg} \mathrm{s}^{-1}\right)\end{array}$ & $\begin{array}{c}v_{\text {out }} \\
\left(\mathrm{km} \mathrm{s}^{-1}\right)\end{array}$ & $\dot{P} /\left(L_{A G N} / \mathrm{c}\right)$ & Refs \\
\hline \multicolumn{5}{c}{ Quasars (X-rays) } \\
\hline IRAS F11119+3257 & 46.2 & 75000 & $1.3_{-0.9}^{+1.7}$ & $\#$ \\
APM 08279+5255 & 47.0 & 60000 & $0.6_{-0.4}^{+1.2}$ & 15 \\
PG 1211+143 & 45.7 & 42000 & $1.6_{-0.8}^{+1.6}$ & 16 \\
HS 1700+6416 & 47.0 & 90000 & $1 \pm 0.2$ & 17 \\
HS 0810+2554 & 46.0 & 90000 & $1.8_{-1.1}^{+1.6}$ & 18 \\
PDS 456 & 47.0 & 75000 & $1.9 \pm 0.8$ & 19 \\
\hline \multicolumn{5}{c}{ ULIRGs (OH) } \\
\hline IRAS F11119+3257 & 46.2 & 1000 & $11.0_{-7.5}^{+14.1}$ & $\#$ \\
IRAS 08572+3915 & 45.7 & 700 & $25_{-18}^{+75}$ & 5 \\
IRAS 13120-5453 & 44.9 & 520 & $18_{-12}^{+50}$ & 5 \\
Mrk 231 & 46.0 & 1000 & $18 \pm 3$ & 7 \\
\hline \multicolumn{5}{c}{ ULIRGs (CO) } \\
\hline IRAS F08572+3915 & 45.7 & 800 & $35_{-22}^{+63}$ & 8 \\
IRAS F10565+2448 & 44.8 & 450 & $40_{-26}^{+72}$ & 8 \\
IRAS 23365+3604 & 44.7 & 450 & $31_{-20}^{+56}$ & 8 \\
Mrk 231 & 46.0 & 1000 & $20 \pm 10$ & 4 \\
\hline
\end{tabular}

The reported velocity $v_{\text {out }}$ refers to the average outflow velocity. The parameters $\dot{P}$ and $L_{A G N} / c$ indicate the momentum flux of the outflow and AGN radiation, respectively. Errors are at the $1 \sigma$ level. The last column indicates the relevant references, $4,5,7,8,15-19$. The parameters of the inner $\mathrm{X}$-ray wind and $\mathrm{OH}$ molecular outflow in IRAS F11119+3257 indicated by \# refer to this work. 